\title{
Place des oncogènes et des facteurs de croissance dans la physiopathologie du psoriasis
}

Le psoriasis cst une dermatose fréquente. Sa nature est le plus souvent bénigne, mais sa chronicité, son étendue, son retentissement psycho-affectif en font une maladie de morbidité non négligeable [1]. Malgré les très nombreux travaux qui se sont intéressés à la physiopathologie de la maladie et qui ont soulevé des hypothèses séduisantes, son étiologie précise demeure, à l'heure actuelle, inconnue.

Le psoriasis associe une hyperprolifération des kératinocytes, une anomalie de leur différenciation et une inflammation dermique importante.

L'cnscmble de ces manifestations témoigne de l'activation simultanée et possiblement réciproque de plusieurs groupes ccllulaires.

Dans les hypothèses pathogéniques actuelles, où pourraient intervenir les oncogènes cellulaires ?

L'hyperprolifération épidermique psoriasique pourrait trouver son origine dans des facteurs extra-kératinocytaires ou intra-kératinocytaires.

\section{- Au niveau du kératinocyte}

Le kératinocyte psoriasique pourrait anormalement synthétiser des facteurs mitogènes.

Les premiers candidats susceptibles d'intervenir à ce niveau sont représentés par les facteurs de croissance et leurs récepteurs, qu'ils appartiennent ou non à la famille des oncogènes cellulaires. Parmi eux, les facteurs mis en jeu lors de la cicatrisation cutanée sont les premiers visés. En effet, on a montré que le PDGF, l'EGF, le TGF $\alpha$ et $\beta$, le bFGF, les cytokines IL-1, IL-6, IL-8 et l'insuline growth factor jouent un rôle important dans les phénomènes de riasis est comparé par certains auteurs à une "cicatrisation qui n'en finit pas " [11]. On peut également envisager que le kératinocyte psoriasique réponde anormalement aux signaux mitogènes par anomalie des systèmes de transduction cellulaire. Parmi ces derniers, les oncogènes cellulaires codant pour des seconds messagers comme les oncogènes $c$-ras, $c$-mos et $c$ raf pourraient être incriminés. Enfin, les oncogènes nucléaires ayant des activités d'activateurs transcriptionnels pourraicnt être responsables d'un déréglement de la croissance cellulaire et/ou de la différenciation à point de départ nucléaire.

\section{- Facteurs extra-kératinocytaires}

Dans cette hypothèse, les anomalies proposées ci-dessus n'intéresseraient plus le kératinocyte mais diverses cellules dont le rôle dans la pathogénie du psoriasis a été soulevé : les fibroblastes, les polynucléaires, les cellules de Langerhans, les cellules endothéliales, les cellules immunitaires (en particulier les lymphocytes $\mathrm{T}$ ). Le kératinocyte psoriasique, intrinsèquement normal, répondrait alors à des signaux extérieurs de prolifération. Parmi ces signaux, certains pourraient appartenir à la famille des oncogènes cellulaires, ou agir sur les kératinocytes ou les cellules environnantes en activant certains oncogènes cellulaires.

État actuel des connaissances sur le rôle des oncogènes cellulaires dans le psoriasis

Aucun modèle in vitro fiable n'existant pour l'instant, l'essentiel des travaux a été réalisé sur des biopsies de peaux lésées psoriasiques.

- Parmi les facteurs de croissance et leurs récepteurs, on a montré une augmentation de l'expression du récepteur du PDGF par les cellules dermiques [3] et une anomalie de régulation de l'expression du récepteur de l'EGF (c-erb B1) dans les couches basales et suprabasales épidermiques [12, 13]. Cependant, Elder et al. n'ont pas retrouvé d'augmentation de l'ARNm de c-erb B1 dans la peau psoriasique [14]. Curieusement, un traitement des lésions psoriasiques par de l'EGF provoque une diminution du phénotype psoriasique par régulation négative de l'expression de c-erbB1 [15]. - De même, une expression élevée du récepteur de l'IGF-I (insulin growth factor I) a été montrée dans l'épiderme psoriasique [16].

Des résultats intéressants ont été rapportés pour divers facteurs de croissance : - l'expression du TGF $\alpha$ est augmentée. Les taux d'ARNm et de protéine sont significativement plus élevés que ceux observés dans la peau normale [14] ;

- l'expression des interleukines 6 et 8 est également plus importante dans la peau lésée que dans la peau normale. L'IL-8 ou neutrophil activating peptide (NAP) est assimilé à une classe d'oncogènes dont se rapproche l'oncogènes gro. Récemment, une augmentation de l'expression de l'oncogène gro a été rapportée dans le psoriasis [17]. Ces facteurs pourraient également intervenir dans la composante inflammatoire de la maladie ;

- il existe une augmentation de l'expression du TNF $\alpha$ dans le derme psoriasique [18]. Cela pourrait expliquer l'hyperexpression d'IL-8 dans la maladie, puisque le $\mathrm{TNF} \alpha$ est connu pour être un inducteur de cette molécule. 
Des anomalies des systèmes intracellulaires de transduction ont depuis longtemps attiré l'attention dans le psoriasis. Des anomalies du système adénylate cyclase, dont certains composants pourraient être rangés parmi des oncogènes potentiels, comme la sous-unité $\alpha$ de la protéine GS, ont été rapportés. Kobayashi a rapporté une distribution anormale de la protéine $\mathrm{p} 21^{\text {ras }}$ dans la peau psoriasique où, contrairement à son expression limitée à la couche basale de l'épiderme normal, elle est retrouvée exprimée sur toute la hauteur de l'épiderme psoriasique [19]. Mordovstek et al. ont également rapporté une augmentation des quantités d'ARNm de Ki-ras dans l'épiderme psoriasique [20], ce qui n'a pas été confirmé par d'autres auteurs. En revanche, aucune modification de la structure de la protéine p21 $1^{\text {ras }}$ n'a été démontrée dans le psoriasis [21]. Le petit nombre d'études consacrées à cette classe d'oncogènes n'exclut pas l'intervention de certains d'entre eux dans la physiopathologie de la maladie. Nous nous sommes plus particulièrement intéressés aux proto-oncogènes $c$-fos et $c$-jun qui codent pour des modulateurs transcriptionnels. Il a été montré que leur expression est élevée dans la peau humaine normale, en particulier dans le compartiment prolifératif de l'épiderme [22]. Nous avons trouvé des taux d'ARNm et de protéines significativement abaissés dans l'épiderme lésionnel psoriasique, suggérant que ces proto-oncogènes jouent plus un rôle dans le contrôle de la différenciation que dans celui de la prolifération des kératinocytes [23]. Par ailleurs, nous avons pu montrer que ces anomalies n'étaient pas spécifiques du psoriasis mais pouvaient être retrouvées dans des dermatoses où les modifications morphologiques sont proches de celles qui sont observées dans le psoriasis, comme l'atopie ou la dermite séborrhéique. Nous avons par ailleurs montré que $c$-fos restait inductible dans l'épiderme psoriasique, ce qui témoigne du bon fonctionnement de son promoteur. Par ailleurs, dans les kératinocytes, l'expression de c-fos est inductible par le calcium, agent induisant la différenciation de ces cellules. Nous avons également recherché s'il existait une activation de certains oncogènes dans les fibroblastes psoriasiques, mais nous n'avons pas retrouvé de différence par rapport à leur expression dans des fibroblastes normaux [24]. Cependant, des travaux récents menés par Casado et al. ont montré une augmentation anormale de l'expression de c-myc sous l'influence d'un analogue de la vitamine $\mathrm{D}$ dans les fibroblastes psoriasiques (communication personnelle). Par ailleurs, une plus grande sensibilité des fibroblastes psoriasiques à l'effet chimiotactique et mitogénique du PDGF sous l'influence de l'acide rétinoïque a été récemment rapportée [25]. Ainsi, un certain nombre d'anomalies de l'expression de divers oncogènes ont été retrouvées dans le psoriasis. L'augmentation de l'expression de certains facteurs de croissance et de leurs récepteurs ainsi qu'une anomalie des systèmes de transduction intracellulaire pourraient expliquer l'hypercanthose épidermique ; la diminution de l'expression d'oncogènes comme $c$-fos et c-jun pourrait expliquer la mauvaise différenciation observée dans la peau lésionnelle psoriasique. Néanmoins, il est à l'heure actuelle impossible de distinguer, parmi l'ensemble des anomalies observées, celles qui pourraient jouer un rôle dans la pathogénie de l'affection et celles qui ne sont que réactionnelles. La situation est d'ailleurs d'autant plus confuse qu'il n'existe pas vraiment d'anomalies strictement spécifiques à la maladie. Par ailleurs, de nombreux oncogènes restent à étudier, si bien qu'il demeure possible que de telles études débouchent sur la caractérisation d'anomalies propres à cette maladie. Enfin, il est de plus clairement démontré que les oncogènes cellulaires appartiennent à des superfamilles de gènes. Au sein d'une même famille, les divers membres peuvent agir en synergie ou au contraire exercer un rétrocontrôle négatif sur leur propre activité. Le système des proto-oncogènes cellulaires est donc d'une très grande complexité, et une analyse plus fine des divers membres de chaque famille pourrait apporter des informations plus précises sur le rôle de ces gènes dans cette maladie. L'affinement des méthodes de culture ex vivo fournira peut-être des modèles pathologiques plus fiables. Les souris transgéniques sont également des modèles intéressants. Ainsi Roop et al. ont rapporté leurs résultats utilisant des sou- ris transgéniques pour le $\mathrm{TGF} \alpha$ (communication orale ESDR, Londres, 1992) ( $m / s n^{\circ} 10$, vol. 8, p. 1123). Ces animaux font une dermatose avec hyperkératose proche du psoriasis, mais où manquent les phénomènes inflammatoires propres à la maladie. D'autres modèles d'animaux transgéniques pour divers facteurs de croissance ou oncogènes cellulaires sont en cours dans diverses équipes.

Enfin, le rôle d'une autre classe de gènes plus récemment identifiée est à explorer : celles des anti-oncogènes. Ces derniers exercent un contrôle négatif sur la prolifération cellulaire et sont impliqués dans de nombreux processus tumoraux associés à leur inactivation. Des travaux récents ont suggéré que le gène p53 pouvait être impliqué dans le psoriasis. Néanmoins, dans notre laboratoire, une étude par PCR suivie d'une analyse du polymorphisme conformationnel des simples brins (ou PCR-SSCP) [26] et par immunohistochimie, ne nous a pas permis de retrouver de mutation du gène p53 (au moins dans les domaines hyperconservés, qui sont les principales cibles mutationnelles) ni d'anomalie de son expression dans l'épiderme lésionnel psoriasique [27]. D'autres anti-oncogènes restent à étudier, d'autant qu'il est possible de concevoir une prédisposition génétique de la maladie, transmissible à la descendance, et qui pourrait être démasquée sous l'influence de divers facteurs de l'environnement

\section{TIRÉS A PART}

N. Basset-Sćguin 
1. Guilhou JJ. Psoriasis. Aspects cliniques et diagnostic différentiel. Éditions Techniques. Encycl Mód Chir (Panis-France), Dermatologie 12310 A10, 1992, 8 p.

2. Guilhou .JJ. Psoriasis. Histopathologie, histogenèse ct étiopathogénie. Éditions Techniques. Encycl Méd Chir (Paris-France), Dermalologie, 12310 A20, 1992, 6 p.

3. Krane JF, Murphy DP, Gottlieb AB, Carter DM, Hart CE, Krucger JG. Increased dermal expression of platclet derived growth factor receptors in growth-activated skin wounds and psoriasis. J Invest Dermalol 1991 ; 96 : 983-6.

4. Rosen EM, Goldberg ID, Liu D, Setter E, Donovan MA, Bhargava M, Reiss $M$, Kacinski BM. Tumor necrosis factor stimulates epithelial tumor ccll motility. Cancer Res $1991 ; 51$ : 5315-21.

5. Jones SC, Curtsinger LJ, Whalen JD, Piestch JD, Ackerman D, Brown GL, Schultz GS. Effect of topical recombinant TGF b on healing of partial thickness injuries. J Surg Res 1991 ; 51 : 344-52.

6. Whitby DJ, Ferguson MW. Immunohistochemical localization of growth factors in fetal wound healing. Dev Biol 1991; 147 : 207-15.

7. Mertz PM, Sauder DL, Davis SC, Kilian PL, Herron AJ, Eaglstein WH. IL1 as a potent inducer of wound rc-cpithclization. Prog Clin Biol Res 1991； 365 : 473-80.

8. Klingbeil CK, Cesar LB, Fiddes JC. Basic fibroblast growth factor accelerates tissue repair in models of impaircd wound healing. Prog Clin Biol Res 1991; 365 : 443-58.

9. Kiyohara Y, Komada F, Iwakawa S, Hirai M, Fuwa T, Okumura K. Improvement in wound healing by epidermal growth factor ointment. J Pharmacobiodyn 1991; 14 : 47-52.

10. Schultz G, Rotatori DS, Clark W. EGF and TGF $\alpha$ in wound healing and repair. $J$ Cell Biochem 1991; 45 : 346-52.

11. Dubcrtret L, Touraine R. Lc psoriasis une cicatrisation qui n'en finit pas. Pour la Science 1987 ; 118 : 38-48.

12. Amagai M, Ozawa S, Ueda M, Nischikawa T, Abe O, Shimizu N. Distribution of EGF receptor expressing and DNA replicating cells in psoriasis and bowen disease. $\mathrm{Br}$ J Dermatol 1988 ; 119 : 661-8.

13. Nanney LB, Stoscheck CM, Magid M, King LE. Altered (125)I epidermal growth factor binding and receptor distribution in psoriasis. J Invest Dermalol 1986 ; 86 : 260-5.

14. Elder JT, Tavakkol A, Klein SB, Zigler ME, Wicha M, Voorhees IJ. Proto-oncogene expression in normal and psoriatic skin. $J$ Invest Dermalol $1990 ; 94$ : 19-25.
15. Nanncy LB, Yates RA, King LE. Modulation of cpidermal growth factor receptors in psoriatic lesions during treatment with topical EGF. J Invest Dermatol 1992 ; 98 : 296-301.

16. Kranc JF, Gottlieb AB, Carter DM, Krucger JG. The insulin-like growth factor receptor is ovcrexpressed in psoriatic cpidermis but is differentially regulated from the epidermal growth factor receptor. J Exp Med $1992 ; 175: 1081-90$.

17. Scroder JM, Gregory H, Young J, Christophers E. Ncutrophil-activating protcins in psoriasis. J Invest Dermalol 1992 ; 98 : 241-7.

18. Nickoloff BJ, Karabin GD, Barker JN Griffiths CE, Sarma V, Mitra RS, Elder JT, Kunkel SL, Dixit VM. Cellular localization of interlcukin 8 and its inducer, tumor necrosis factor alpha in psoriasis. Am J Pathol 1991 ; $138: 129-40$.

19. Kobayashi $H$, Yasuda $H$, Ohkawara A, Dosaka H, Oda A, Ogiso Y, Kuzumaki N. Enhanced expression of ras gene products in psoriatic epidermis. Arch Dermalol Res 1988 ; 280 : 259-63.

20. Mordovtscv VN, Starkov IV, Zabarovsky ER, Kissclev LL. Expression of the protooncogenes in psoriatic lesions. Arch Dermatol Res 1988 ; $280: 8-11$.

21. Takahashi $\mathrm{H}$, Lizuka $H$, Katagiri $M$. No evidence for the mutation of ras gene in psoriatic epidernnis. Arch Dermalol Res $1990 ; 282$ : 8-11.

22. Basset-Séguin $\mathrm{N}$, Escot $\mathrm{C}$, Blanchard JM, Kérai C, Verrier B, Mion H, Guilhou JJ. High levels of c-fos proto-oncogene expression in normal human skin. J Invest Dermatol 1990 ; $94: 418-22$

23. Basset-Séguin N, Escot C, Moles JP, Blanchard JM, Kcrai C, Guilhou JJ. c-fos and cjun proto-oncogene expression is decreased in psoriasis : an in situ quantitative analysis. $J$ Invest Dermalol $1991 ; 97: 672-8$.

24. Basset-Sćguin N, Vie A, Blanchard JM, Guilhou IJ. Expression of c-myc, c-myb, cerb A et c-H-ras oncogene mRNAs in fibroblasts cultured from psoriatic patients. Dermatologica $1987 ; 175$ : 296-9.

25. Raynaud F, Gerbaud P, Gu XF, Donnadicu M, Evain-Brion D. Effect of retinoic acid on platelet-derived growth factor (PDGF) bioactility and type B PDGF receptors in normal and psoriatic human fibroblasts. $J$ Invest Dermalol 1991; $96: 111-5$.

26. Dreyfus JC, Akli S, Poenaru L. Maladies de Tay-Sachs et Sandhoff : les déficits en $\beta$-hexosaminidases, modèles de maladies des lysosomes. médecine/scinces $1992 ; 8: 797-804$.

27. Molès JP, Theillct $C$, Basset-Sćguin $N$, Guilhou JJ. Mutation of the human suppressor gene TP53 is not detected in a psoriatic skin. J Invest Dermalol 1993 (sous presse). 